\title{
Compatible Deductive Systems of Pulexes
}

\author{
Shokoofeh Ghorbani \\ Department of Mathematics, Shahid Bahonar University of Kerman, Kerman, Iran \\ Correspondence should be addressed to Shokoofeh Ghorbani; sh.ghorbani@uk.ac.ir
}

Received 7 February 2014; Accepted 2 May 2014; Published 19 May 2014

Academic Editor: Aloys Krieg

Copyright (C) 2014 Shokoofeh Ghorbani. This is an open access article distributed under the Creative Commons Attribution License, which permits unrestricted use, distribution, and reproduction in any medium, provided the original work is properly cited.

\begin{abstract}
The notion of (compatible) deductive system of a pulex is defined and some properties of deductive systems are investigated. We also define a congruence relation on a pulex and show that there is a bijective correspondence between the compatible deductive systems and the congruence relations. We define the quotient algebra induced by a compatible deductive system and study its properties.
\end{abstract}

\section{Introduction}

Imai and Iséki [1] introduced the concept of BCK-algebra as a generalization of notions of set difference operation and propositional calculus. The notion of pseudo-BCK algebra was introduced by Georgescu and Iorgulescu [2] as generalization of BCK-algebras not assuming commutativity.

Hájek [3] introduced the concept of BL-algebras as the general semantics of basic fuzzy logic (BL-logic). Iorgulescu studied BCK-algebras and their relation to BL-algebras [4, 5]. BL-algebra has been generalized in different ways [68]. Hájek in [9] introduced flea-algebra as a generalization of BL-algebra. He proved that the implication reduct of a flea-algebra is a pseudo-BCK algebra with three additional conditions. The pseudo-BCK algebra with these conditions is called pulex.

In this section some preliminary definitions and theorems are stated. In Section 2, we introduce the notions of deductive system, lattice filter, and subalgebra of pulexes and obtain some properties which are not true in a pseudoBCK lattice in general. In Section 3, we define concepts of compatible deductive system and congruence relation on a pulex and show that there is a correspondence between the set of all compatible deductive systems of a pulex and the set of all congruence relations on a pulex which is not true in a pseudo-BCK lattice. After that we prove that the quotient algebra defined by a congruence relation is a pulex and we obtain some related results.
Definition 1 (see [10]). A pseudo- $B C K$ algebra is a structure $\mathscr{A}=(A, \leq, \rightarrow, \rightsquigarrow, 1)$, where $(A, \leq)$ is a poset with the greatest element 1 and $\rightarrow, \rightsquigarrow$ are binary operations on $A$ such that, for all $x, y, z \in A$, we have

$$
\begin{aligned}
& \text { (1) } x \rightarrow y \leq(y \rightarrow z) \rightsquigarrow(x \rightarrow z), x \rightsquigarrow y \leq(y \rightsquigarrow \\
& z) \rightarrow(x \rightsquigarrow z), \\
& \text { (2) } x \leq(x \rightarrow y) \rightsquigarrow y, x \leq(x \rightsquigarrow y) \rightarrow y, \\
& \text { (3) } x \leq y \text { iff } x \rightarrow y=1 \text { iff } x \rightsquigarrow y=1 .
\end{aligned}
$$

Theorem 2 (see [11]). An algebra $\mathscr{A}=(A, \rightarrow, \rightsquigarrow, 1)$ of type $(2,2,0)$ is a pseudo-BCK algebra if and only if it satisfies the following:

(1) $(x \rightarrow y) \rightsquigarrow[(y \rightarrow z) \rightsquigarrow(x \rightarrow z)]=1$ and $(x \rightsquigarrow y) \rightarrow[(y \rightsquigarrow z) \rightarrow(x \rightsquigarrow z)]=1$;

(2) $1 \rightarrow x=x, 1 \leadsto x=x$;

(3) $x \rightarrow 1=1, x \rightsquigarrow 1=1$;

(4) $x \rightarrow y=1$ and $y \rightarrow x=1$ implies $x=y$, the same for $\rightsquigarrow$.

Definition 3 (see [11]). If the partial order $\leq$ of a pseudo-BCK algebra $\mathscr{A}=(A, \leq, \rightarrow, \rightsquigarrow, 1)$ is a lattice order, with the lattice operations $\wedge$ and $\vee$, then $\mathscr{A}$ is said to be a pseudo-BCK lattice and it will be denoted by $\mathscr{A}=(A, \wedge, \vee, \rightarrow, \rightsquigarrow, 1)$. 
Example 4. Let $A=\{0, a, b, c, d, 1\}$ such that $0<a<b<$ $c<1,0<d<c<1$, and $a, b$ are incomparable with $d$. The operations $\rightarrow$ and $\leadsto$ are given as follows:

\begin{tabular}{c|cccccc}
$\longrightarrow$ & 0 & $a$ & $b$ & $c$ & $d$ & 1 \\
\hline 0 & 1 & 1 & 1 & 1 & 1 & 1 \\
$a$ & 0 & 1 & 1 & 1 & $c$ & 1 \\
$b$ & 0 & $b$ & 1 & 1 & $c$ & 1 \\
$c$ & 0 & $b$ & $b$ & 1 & $c$ & 1 \\
$d$ & 0 & $b$ & $b$ & 1 & 1 & 1 \\
1 & 0 & $a$ & $b$ & $c$ & $d$ & 1
\end{tabular}

\begin{tabular}{c|cccccc}
$\rightsquigarrow$ & 0 & $a$ & $b$ & $c$ & $d$ & 1 \\
\hline 0 & 1 & 1 & 1 & 1 & 1 & 1 \\
$a$ & 0 & 1 & 1 & 1 & $c$ & 1 \\
$b$ & 0 & $c$ & 1 & 1 & $c$ & 1 \\
$c$ & 0 & $a$ & $b$ & 1 & $c$ & 1 \\
$d$ & 0 & $a$ & $b$ & 1 & 1 & 1 \\
1 & 0 & $a$ & $b$ & $c$ & $d$ & 1
\end{tabular}

Then $\mathscr{A}=(A, \wedge, \vee \rightarrow, \rightsquigarrow, 1)$ is a pseudo-BCK lattice.

Definition 5 (see [9]). A pulex is a structure $P=(P, \wedge, \vee, \rightarrow$ $, \rightsquigarrow, 1)$ such that

(1) $P=(P, \wedge, \vee, \rightarrow, \rightsquigarrow, 1)$ is a pseudo-BCK lattice;

(2) $(x \rightarrow y) \rightarrow z \leq[(y \rightarrow x) \rightarrow z] \rightarrow z$, the same for $n$;

(3) $x \vee y=[(x \rightarrow y) \rightsquigarrow y] \wedge[(y \rightarrow x) \rightsquigarrow x]=[(x \rightsquigarrow$ $y) \rightarrow y] \wedge[(y \rightsquigarrow x) \rightarrow x]$

(4) $(x \rightarrow y) \rightarrow(x \rightarrow(x \wedge y))=1$, the same for $\rightsquigarrow$.

Remark 6. We see that the pseudo-BCK lattice of Example 4 is not a pulex because $(b \rightarrow d) \rightarrow(b \rightarrow(b \wedge d))=0 \neq 1$.

Definition 7 (see [9]). A flea-algebra is a structure $(F, \wedge, \vee, *, \rightarrow, \rightsquigarrow, 1)$ where

(1) $(F, \wedge, \vee, 1)$ is a lattice with the greatest element 1 ;

(2) * is a binary associative operation with 1 as a bothside unit;

(3) $x * y \leq z$ iff $x \leq y \rightarrow z$ iff $y \leq x \leadsto z$ (residuation);

(4) $(x \rightarrow y) \vee(y \rightarrow x)=1,(x \rightsquigarrow y) \vee(y \rightsquigarrow x)=1$ (prelinearity).

Proposition 8 (see [9]). Let $(F, \wedge, \vee, *, \rightarrow, \rightsquigarrow, 1)$ be a fleaalgebra. Then $(F, \wedge, \vee, \rightarrow, \rightsquigarrow, 1)$ is a pulex.

Proposition 9 (see $[9,10])$. In any pulex, the following rules hold:

(1) $x \rightarrow[(x \rightsquigarrow y) \rightarrow y]=1$,

(2) $x \rightsquigarrow[(x \rightarrow y) \leadsto y]=1$,

(3) $x \rightarrow x=x \rightsquigarrow x=1$,

(4) $x \rightarrow y=1$ iff $x \rightsquigarrow y=1$,

(5) $x \leq y$ implies that $z \rightarrow x \leq z \rightarrow y$ and $z \backsim x \leq$ $z \rightsquigarrow y$,

(6) $x \leq y$ implies that $y \rightarrow z \leq x \rightarrow z$ and $y \backsim z \leq$ $x \rightsquigarrow z$,

(7) $x \rightsquigarrow(y \rightarrow z)=y \rightarrow(x \rightsquigarrow z)$,

(8) $x \leq y \rightarrow z$ iff $y \leq x \rightsquigarrow z$,

(9) $x \leq y \rightarrow x$ and $x \leq y \rightsquigarrow x$,

(10) $((x \rightsquigarrow y) \rightarrow y) \rightsquigarrow y=x \rightsquigarrow y$ and $((x \rightarrow y) \rightsquigarrow$ $y) \rightarrow y=x \rightarrow y$,
(11) $x \rightarrow y \leq(z \rightarrow x) \rightarrow(z \rightarrow y)$ and $x \rightsquigarrow y \leq(z \rightsquigarrow$ $x) \rightsquigarrow(z \rightsquigarrow y)$,

(12) $((x \rightarrow y) \rightsquigarrow y) \rightsquigarrow y \leq x \rightsquigarrow y$ and $((x \rightsquigarrow y) \rightarrow$ $y) \rightarrow y \leq x \rightarrow y$.

Proposition 10. In every pulex $P=(P, \wedge, \vee, \rightarrow, \backsim, 1)$, the following relations hold for all $a, b, c \in P$ :

$$
\begin{gathered}
a \longrightarrow b \leq(a \wedge c) \longrightarrow(b \wedge c), \\
a \rightsquigarrow b \leq(a \wedge c) \rightsquigarrow(b \wedge c) .
\end{gathered}
$$

Proof. Since $a \wedge c \leq a$, then $a \rightarrow b \leq(a \wedge c) \rightarrow b$ by Proposition 9 part (6). We have $(a \wedge c) \rightarrow b \leq(a \wedge c) \rightarrow$ $(a \wedge b \wedge c)$ by Definition 5 part (4). Since $a \wedge b \wedge c \leq b$, we get that $(a \wedge c) \rightarrow(a \wedge b \wedge c) \leq(a \wedge c) \rightarrow(b \wedge c)$ by Proposition 9 part (5). Therefore $a \rightarrow b \leq(a \wedge c) \rightarrow(b \wedge c)$.

Similarly, we can show $a \rightsquigarrow b \leq(a \wedge c) \rightsquigarrow(b \wedge c)$.

Proposition 11. Let $P=(P, \wedge, \vee, \rightarrow, \rightsquigarrow, 1)$ be a pulex. Then the following relations hold:

(1) $(a \vee b) \rightarrow c=(a \rightarrow c) \wedge(b \rightarrow c)$ and $(a \vee b) \leadsto c=$ $(a \rightsquigarrow c) \wedge(b \leadsto c)$,

(2) $a \rightarrow b \leq(a \vee c) \rightarrow(b \vee c)$ and $a \rightsquigarrow b \leq(a \vee c) \rightsquigarrow$ $(b \vee c)$, for all $a, b, c \in P$.

Proof. (1) Since $a, b \leq a \vee b$, by Proposition 9 part (6),

$$
(a \vee b) \longrightarrow c \leq a \longrightarrow c, \quad(a \vee b) \longrightarrow c \leq b \longrightarrow c .
$$

Hence $(a \vee b) \rightarrow c \leq(a \rightarrow c) \wedge(b \rightarrow c)$. On the other hand, we have

$$
\begin{aligned}
& (a \longrightarrow c) \wedge(b \longrightarrow c) \leq a \longrightarrow c \\
& (a \longrightarrow c) \wedge(b \longrightarrow c) \leq b \longrightarrow c .
\end{aligned}
$$

By Proposition 9 parts (2), (6),

$$
\begin{aligned}
& a \leq(a \longrightarrow c) \leadsto c \leq[(a \longrightarrow c) \wedge(b \longrightarrow c)] \leadsto c, \\
& b \leq(b \longrightarrow c) \leadsto c \leq[(a \longrightarrow c) \wedge(b \longrightarrow c)] \leadsto c .
\end{aligned}
$$

Therefore $a \vee b \leq[(a \rightarrow c) \wedge(b \rightarrow c)] \leadsto c$. By Proposition 9 parts (1), (6),

$$
\begin{aligned}
(a \longrightarrow c) \wedge(b \longrightarrow c) & \leq[(a \longrightarrow c) \wedge((b \longrightarrow c)) \leadsto c] \longrightarrow c \\
& \leq a \vee b \longrightarrow c .
\end{aligned}
$$

(2) By part $(1),(a \vee c) \rightarrow(b \vee c)=(a \rightarrow(b \vee c)) \wedge$ $(b \rightarrow(b \vee c))=(a \rightarrow(b \vee c))$. Since $b \leq b \vee c$, we have $a \rightarrow(b \vee c) \geq a \rightarrow b$ by Proposition 9 part (5). Thus $a \rightarrow$ $b \leq(a \vee c) \rightarrow(b \vee c)$

\section{Deductive Systems}

In this section, we introduce and study deductive systems of a pulex. From logical point of view, deductive systems correspond to the sets of formulas which are closed under the inference rule modus ponens. In what follows, we denote a pulex $P=(P, \wedge, \vee, \rightarrow, \rightsquigarrow, 1)$ by $P$. 
Definition 12. Let $F$ be a subset of a pulex $P$. We say that $F$ is a deductive system if

(1) $1 \in F$, and

(2) if $a, a \rightarrow b \in F$, then $b \in F$ for all $a, b \in P$.

Example 13. Let $P$ be the pulex with the universe $\{0, a, b, c, 1\}$ such that $0<a<b, c<1$, and $b, c$ are incomparable. The operations $\rightarrow$ and $\leadsto$ are given by the tables below:

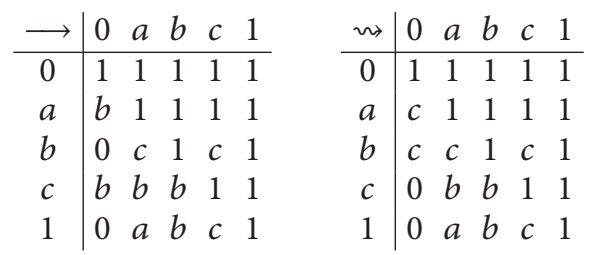

$F_{1}=\{c, 1\}$ and $F_{2}=\{b, 1\}$ are deductive systems of $P$.

Definition 14. Let $P$ be a pulex, and let $F$ be a nonempty subset of $P$. We say that $F$ is a lattice filter of $P$ if

(1) $a \wedge b \in F$ for all $a, b \in F$, and

(2) if $a \in F, b \in P$, and $a \leq b$, then $b \in F$.

Deductive systems of a pseudo-BCK lattice may not be a lattice filter. For example, consider deductive system $F=$ $\{a, b, c, d, 1\}$ in Example 4. Since $a, d \in F$ and $a \wedge d=0 \notin$ $F$, then $F$ is not a lattice filter. In the following, we study the relationship between deductive systems and lattice filters of a pulex.

Theorem 15. Every deductive system of a pulex $P$ is a lattice filter of $P$.

Proof. Let $F$ be a deductive system of a pulex $P$. Since $1 \in F$, then $F \neq \emptyset$.

(i) Let $a \in F$ and $b \in P$ such that $a \leq b$. So we have $a \rightarrow b=1 \in F$. Since $F$ is a deductive system, then we have $b \in F$.

(ii) Let $a, b \in F$. By part (9) of Proposition 9, $a \leq b \rightarrow a$ and then $b \rightarrow a \in F$ by part (i). Since $P$ is a pulex, then $(b \rightarrow a) \rightarrow(b \rightarrow(a \wedge b))=1 \in F . F$ is a deductive system, so $b \rightarrow(a \wedge b) \in F$ and then $a \wedge b \in F$.

Remark 16. The converse of Theorem 15 may not be true. Consider Example 13. $F=\{a, b, 1\}$ is a lattice filter of $P$, but it is not a deductive system because $a \in F$ and $a \rightarrow 0=b \in F$, but $0 \notin F$. Hence the converse of the above theorem is not true in a pseudo-BCK lattice too.

Theorem 17. Let $P$ be a pulex. Then $F \subseteq P$ with $1 \in F$ is a deductive system of $P$ if and only if it satisfies the following condition:

$$
\text { if } a, a \rightsquigarrow b \in F \text {, then } b \in F \quad \forall a, b \in P \text {. }
$$

Proof. See Lemma 2.1.3 in [5].
It is easy to verify that the intersection of deductive systems of a pulex $P$ is a deductive system of $P$.

Definition 18. Let $X$ be a subset of a pulex $P$. The smallest deductive system of $P$ containing $X$ (i.e., the intersection of all deductive systems of $P$ containing $X$ ) is called the deductive system generated by $X$ and will be denoted by $[X)$. If $X=$ $\{x\}$, then $[\{x\})$ is written as $[x)$ and is called the principal deductive system generated by $x$.

For convenience, we will write $x \rightarrow{ }^{0} a=x \rightsquigarrow^{0} a=a$ and

$$
\begin{array}{r}
x \longrightarrow{ }^{n} a=x \longrightarrow(\cdots \longrightarrow(x \longrightarrow a) \cdots), \\
x n^{n} a=x \rightsquigarrow(\cdots \rightsquigarrow(x \rightsquigarrow a) \cdots),
\end{array}
$$

where $n \in \aleph$ indicating the number of occurrence of $x$.

Theorem 19 (see [5]). Let $P$ be a pulex, and let $X$ be a nonempty subset of $P$ and $x, y \in P$. Then

(1) $[X)=\left\{a \in P: x_{1} \rightarrow\left(\cdots \rightarrow\left(x_{n} \rightarrow a\right) \cdots\right)=1\right.$ for some $\left.x_{1} \cdots x_{n} \in X, n \in \aleph\right\}$;

(2) $[x)=\left\{a \in P: x \rightarrow{ }^{n} a=1\right.$ for some $\left.n \in \mathcal{N} \cup\{0\}\right\}$;

(3) $x \leq y$ implies $[y] \subseteq[x)$;

(4) $[x) \cap[y]=[x \vee y)$.

The set of all deductive systems of a pulex $P$ is an algebraic lattice whose compact elements are precisely the finitely generated deductive systems $P$.

Definition 20. A subset $S$ of a pulex $P$ is called subalgebra of $P$ if the constant 1 is in $S$ and $S$ itself forms a pulex under operations of $P$.

Proposition 21. A nonempty subset $S$ of a pulex $P$ is a subalgebra of $P$ if and only if $S$ is closed under operations $\wedge$, $\rightarrow, \rightsquigarrow$ on $P$.

Deductive system of a pseudo-BCK lattice may not be a subalgebra. Consider deductive system $F=\{a, b, c, d, 1\}$ of $A$ in Example 4. We can show that it is not a subalgebra of $A$. In the following theorem, we prove that this is true in each pulexes.

Proposition 22. Let $F$ be a deductive system of a pulex $P$. Then $F$ is a subalgebra of $P$.

Proof. Let $F$ be a deductive system of a pulex $P$. Then $F$ is nonempty subset of $P$. By Theorem $15, F$ is closed under $\wedge$. Suppose that $x, y \in F$. By Proposition 9 part (9), we have $x \leq$ $y \rightarrow x$ and $y \leq x \rightarrow y$. By Theorem 15, we get that $y \rightarrow$ $x, x \rightarrow y \in F$; that is, $F$ is closed under $\rightarrow$. Similarly, we can show that $F$ is closed under $\rightsquigarrow$. Hence $F$ is a subalgebra of $P$ by Proposition 21 .

Remark 23. Consider subalgebra $\{a, 1\}$ of $P$ in Example 13 . Then $S$ is not a lattice filter of $P$. By Theorem $15, S$ is not a deductive system of $P$. Therefore the converse of the above proposition may not be true in general. 


\section{Compatible Deductive Systems}

In this section, we introduce the notions of compatible deductive systems and congruence relations of a pulex and study relationship between them.

Definition 24. Let $F$ be a deductive system of a pulex $P$ such that

$$
x \longrightarrow y \in F \quad \text { iff } x \rightsquigarrow y \in F .
$$

Then $F$ is called a compatible deductive system of $P$.

Example 25. Let $P$ be the pulex with the universe $\{0, a, b, c, 1\}$ such that $0<a<b<c<1$. The operations $\rightarrow$ and $\rightsquigarrow$ are given by the tables below:

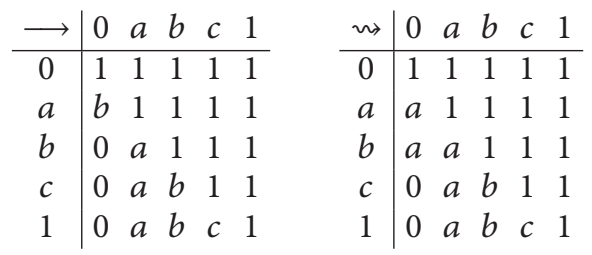

$F_{1}=\{c, 1\}$ is a compatible deductive system of $P$, but the deductive system $F_{2}=\{b, c, 1\}$ of $P$ is not compatible because $a \rightarrow 0=b \in F_{2}$ and $a \rightsquigarrow 0=a \notin F_{2}$.

Definition 26. Let $F$ be a compatible deductive system of a pulex $P$. Define the relation $\theta_{F}$ by

$$
(a, b) \in \theta_{F} \quad \text { iff } a \longrightarrow b \in F, b \longrightarrow a \in F
$$

for all $a, b \in P$.

Theorem 27. $\theta_{F}$ is an equivalence relation on $P$.

Proof. Clearly, $\theta_{F}$ is reflexive and symmetric. Let $(a, b)$, $(b, c) \in \theta_{F}$. Then $a \rightarrow b \in F, b \rightarrow a \in F, c \rightarrow b \in F$, and $b \rightarrow c \in F$. By Proposition 9 part (12),

$$
\begin{aligned}
& c \longrightarrow b \leq(b \longrightarrow a) \leadsto(c \longrightarrow a), \\
& a \longrightarrow b \leq(b \longrightarrow c) \leadsto(a \longrightarrow c) .
\end{aligned}
$$

By Theorem 15, we have $(b \rightarrow a) \leadsto(c \rightarrow a) \in F$ and $(b \rightarrow c) \leadsto(a \rightarrow c) \in F$. Since $F$ is a deductive system and $b \rightarrow a, b \rightarrow c \in F$, then $a \rightarrow c \in F$ and $c \rightarrow a \in F$; that is, $(a, c) \in \theta_{F}$. Hence $\theta_{F}$ is an equivalence relation on $P$.

Definition 28. An equivalence relation $\theta$ on a pulex $P$ is called a congruence relation on $P$ if $(a, b),(c, d) \in \theta$, then

$$
\begin{aligned}
& (a \longrightarrow c, b \longrightarrow d),(a \rightsquigarrow c, b \rightsquigarrow d), \\
& (a \wedge c, b \wedge d),(a \vee c, b \vee d) \in \theta
\end{aligned}
$$

Theorem 29. Let $F$ be a compatible deductive system of a pulex $P$. Then $\theta_{F}$ is a congruence relation on $P$ and is called the congruence relation induced by $F$.
Proof. Suppose that $(a, b),(c, d) \in \theta_{F}$. By Proposition 9 part (11),

$$
\begin{aligned}
& c \longrightarrow d \leq(b \longrightarrow c) \longrightarrow(b \longrightarrow d), \\
& d \longrightarrow c \leq(b \longrightarrow d) \longrightarrow(b \longrightarrow c) .
\end{aligned}
$$

By Theorem 15, we have $(b \rightarrow c) \rightarrow(b \rightarrow d) \in F$ and $(b \rightarrow d) \rightarrow(b \rightarrow c) \in F$ and we have $(b \rightarrow c, b \rightarrow d) \epsilon$ $\theta_{F}$. From

$$
\begin{aligned}
& a \longrightarrow b \leq(b \longrightarrow c) \rightsquigarrow(a \longrightarrow c), \\
& b \longrightarrow a \leq(a \longrightarrow c) \rightsquigarrow(b \longrightarrow c),
\end{aligned}
$$

we obtain $(a \rightarrow c, b \rightarrow c) \in \theta_{F}$. Since $\theta_{F}$ is a transitive relation, we have $(a \rightarrow c, b \rightarrow d) \in \theta_{F}$. Similarly, we can show that $(c \rightarrow a, d \rightarrow b) \in \theta_{F},(c \rightsquigarrow a, d \rightsquigarrow b) \in \theta_{F}$, and $(a \leadsto c, b \leadsto d) \in \theta_{F}$.

By Proposition 10,

$$
\begin{aligned}
& a \longrightarrow b \leq(a \wedge c) \longrightarrow(b \wedge c), \\
& b \longrightarrow a \leq(b \wedge c) \longrightarrow(a \wedge c) .
\end{aligned}
$$

Hence $(a \wedge c) \rightarrow(b \wedge c),(b \wedge c) \rightarrow(a \wedge c) \in F$ by Theorem 15 . Thus $(a \wedge c, b \wedge c) \in \theta_{F}$. Similarly, we can show that $(b \wedge c, b \wedge$ d) $\in \theta_{F}$. Therefore $(a \wedge c, b \wedge d) \in \theta_{F}$.

By Proposition 11 part (2),

$$
\begin{aligned}
& a \longrightarrow b \leq(a \vee c) \longrightarrow(b \vee c), \\
& b \longrightarrow a \leq(b \vee c) \longrightarrow(a \vee c) .
\end{aligned}
$$

Hence $(a \vee c) \rightarrow(b \vee c),(b \vee c) \rightarrow(a \vee c) \in F$ by Theorem 15 Thus $(a \vee c, b \vee c) \in \theta_{F}$. Similarly, we can show that $(b \vee c, b \vee$ d) $\in \theta_{F}$. Therefore $(a \vee c, b \vee d) \in \theta_{F}$.

Theorem 30. Let $\theta$ be a congruence relation on a pulex $P$. Then

$$
F_{\theta}=\{x \in P:(x, 1) \in \theta\}
$$

is a compatible deductive system of $P . F_{\theta}$ is called the compatible deductive system induced by $\theta$.

Proof. Since $\theta$ is reflexive, $1 \in F_{\theta}$. Suppose that $a, a \rightarrow b \in$ $F_{\theta}$. Then $(a, 1),(a \rightarrow b, 1) \in \theta$. Since $\theta$ is a congruence relation, then $(a \rightarrow b, b)=(a \rightarrow b, 1 \rightarrow b) \in \theta$. By transivity, $(1, b) \in \theta$. Hence $b \in F_{\theta}$ and $F_{\theta}$ is a deductive system of $P$.

We will show that $\theta_{F}$ is compatible. Suppose that $a \rightarrow$ $b \in F_{\theta}$, then $(a \rightarrow b, 1) \in \theta$. Since $\theta$ is a congruence relation, then $(((a \rightarrow b) \leadsto b) \rightsquigarrow b, 1)=(((a \rightarrow b) \rightsquigarrow b) \rightsquigarrow$ $b,(1 \rightsquigarrow b) \rightsquigarrow b) \in \theta$. Hence $(a \rightarrow b) \rightsquigarrow b) \rightsquigarrow b \in F_{\theta}$. By Proposition 9 part $(12)((a \rightarrow b) \rightsquigarrow b) \rightsquigarrow b \leq a \rightsquigarrow b$. Since $F_{\theta}$ is a deductive system, $a \rightsquigarrow b \in F_{\theta}$. Similarly, we can show that if $a \rightsquigarrow b \in F_{\theta}$, then $a \rightarrow b \in F_{\theta}$.

Theorem 31. Let $P$ be a pulex. There is a one to one correspondence between the set of all congruence relations on $P$ and the set of all compatible deductive systems of $P$. 
Proof. We will show that $\theta_{F_{\theta}}=\theta$ and $F_{\theta_{F}}=F$,

$$
\begin{aligned}
F_{\theta_{F}} & =\left\{x \in P:(x, 1) \in \theta_{F}\right\} \\
& =\{x \in P: x \longrightarrow 1 \in F, 1 \longrightarrow x \in F\}=F
\end{aligned}
$$

Suppose that $(a, b) \in \theta_{F_{\theta}}$. Then $(a \rightarrow b, 1),(b \rightarrow a, 1) \in \theta$. Since $\theta$ is a congruence relation,

$$
\begin{aligned}
& ((a \longrightarrow b) \rightsquigarrow b, b)=((a \longrightarrow b) \rightsquigarrow b, 1 \rightsquigarrow b) \in \theta, \\
& ((b \longrightarrow a) \rightsquigarrow a, a)=((b \longrightarrow a) \rightsquigarrow a, 1 \rightsquigarrow a) \in \theta .
\end{aligned}
$$

Thus $(a \vee b, a \wedge b)=(((a \rightarrow b) \leadsto b) \wedge((b \rightarrow a) \rightsquigarrow$ $a), a \wedge b) \in \theta$. We have $(a, a \wedge b)=((a \vee b) \wedge a,(a \wedge b) \wedge a) \in \theta$ and $(b, a \wedge b)=((a \vee b) \wedge b,(a \wedge b) \wedge b) \in \theta$. Hence $(a, b) \in \theta$.

Conversely, let $(a, b) \in \theta$. Then

$$
\begin{aligned}
& (1, a \longrightarrow b)=(a \longrightarrow a, a \longrightarrow b) \in \theta, \\
& (b \longrightarrow a, 1)=(b \longrightarrow a, b \longrightarrow b) \in \theta .
\end{aligned}
$$

Thus $a \rightarrow b, b \rightarrow a \in F_{\theta}$; that is, $(a, b) \in \theta_{F_{\theta}}$.

Remark 32. In a pseudo-BCK lattice there is no one-toone correspondence between congruence relations and compatible deductive systems. For example, $F=\{d, c, 1\}$ is a compatible deductive system in Example 4, but $\theta_{F}$ is not a congruence relation on $\mathscr{A}$. Suppose that $\theta_{F}$ is a congruence relation on $\mathscr{A}$. Then $(1, d) \in \theta_{F}$ implies $(b, 0)=(b \wedge 1, b \wedge d) \epsilon$ $\theta_{F}$. Thus $(0,1)=(b \rightarrow 0,0 \rightarrow 0) \in \theta_{F}$; that is, $0=1 \rightarrow 0 \in$ $F$ which is a contradiction.

Definition 33. Let $F$ be a compatible deductive system of a pulex $P$, and let $\theta_{F}$ be the equivalence relation induced by $F$. The set of all equivalence classes $[a]=\left\{b \in P:(a, b) \in \theta_{F}\right\}$ is denoted by $P / F$. On this set define

$$
\begin{aligned}
{[a] \wedge[b] } & =[a \wedge b], \\
{[a] \vee[b] } & =[a \vee b], \\
{[a] \longrightarrow[b] } & =[a \longrightarrow b], \\
{[a] \leadsto[b] } & =[a \leadsto b], \\
1 & =[1]
\end{aligned}
$$

$P / F$ is called the quotient algebra of $P$ with respect to the compatible deductive system $F$.

Theorem 34. Let $F$ be a compatible deductive system of a pulex $P$. Then the quotient algebra $P / F$ is a pulex and is called the quotient pulex.

Proof. Since $\theta_{F}$ is a congruence relation, all the above relations are well defined. It is easy to show that $(P / F, \wedge, \vee, 1)$ is a lattice with the greatest element 1 . Hence we have a relation $\leq$ on $P / F$ defined by

$$
[a] \leq[b] \quad \text { iff }[a] \vee[b]=[b] \text {, }
$$

for all $[a],[b] \in P / F$. By Proposition 11 part (1), $a \rightarrow b=$ $a \vee b \rightarrow b$ and $a \rightsquigarrow b=(a \vee b) \rightsquigarrow b$. It follows that

$$
[a] \leq[b] \quad \text { iff } a \longrightarrow b \in F \text { iff } a \rightsquigarrow b \in F \text {. }
$$

From (24) and (25), we have

$$
\begin{array}{r}
{[a] \leq[b] \quad \text { iff }[a] \vee[b]=[a]} \\
\text { iff }[a] \longrightarrow[b]=1 \\
\text { iff }[a] \leadsto[b]=1,
\end{array}
$$

for all $[a],[b] \in P / F$. Now, it is easy to prove that $(P / F, \wedge, \vee, \rightarrow, \rightsquigarrow, 1)$ is a pulex.

Theorem 35. Let $F$ be a compatible deductive system of pulex $P$, and let $P / F$ be the corresponding quotient algebra. Then the map $f: P \rightarrow P / F$ defined by $f(x)=[x]$ for all $x \in P$ is a homomorphism and its $\operatorname{Ker}(f)=F$ where $\operatorname{Ker}(f)=\{x \in P$ : $f(x)=1\}$.

Proof. The proof is straightforward.

Theorem 36. Let $F$ be a compatible deductive system of a pulex $P$. There is a bijection from the set $D(P, F)$ of all deductive systems of $P$ containing $F$ to the set of $D(P / F)$ of all deductive systems of $P / F$.

Proof. It is easy to prove that

(i) if $J$ is a deductive system of $P$ such that $F \subseteq J$, then $F$ is a compatible deductive of the subalgebra $J$ of $P$ and the quotient pulex $J / F$ is a compatible deductive system of $P / F$;

(ii) if $K$ is a deductive system of $P / F$, then $J=\{a:[a] \epsilon$ $K\}$ is a deductive system of $P$ and moreover $F \subseteq J$ and $K=J / F$.

Now, define $f: D(P, F) \rightarrow D(P / F)$ by $f(J)=J / F$. By (i) $f$ is well define and by (ii) $f$ is onto. We will show that $f$ is one to one. Suppose that $J_{1}, J_{2} \in D(P, F)$ such that $J_{1} \neq J_{2}$. Without loss of generality, we assume that $J_{1} \nsubseteq J_{2}$. Then there exists $x \in J_{1} \backslash J_{2}$. If $f\left(J_{1}\right)=f\left(J_{2}\right)$, then $[x] \in f\left(J_{2}\right)$. So there exists $y \in J_{2}$ such that $[x]=[y]$; that is, $x \rightarrow y \in F$ and $y \rightarrow x \in F$. Since $F \subseteq J_{2}$, then $y \rightarrow x \in J_{2}$. Thus $x \in J_{2}$ which is a contradiction. Hence $f\left(J_{1}\right) \neq f\left(J_{2}\right)$ and $f$ is one to one.

\section{Conclusion}

We introduced the notions of compatible deductive systems and congruence relations on a pulex and obtained some related results. Then we studied the relation between them. We used the compatible deductive systems to define the quotient pulex.

\section{Conflict of Interests}

The author declares that there is no conflict of interests regarding the publication of this paper. 


\section{References}

[1] Y. Imai and K. Iséki, "On axiom systems of propositional calculi. XIV," Proceedings of the Japan Academy, vol. 42, pp. 19-22, 1966.

[2] G. Georgescu and A. Iorgulescu, "Pseudo-BCK algebras: an extension of BCK algebras," in Combinatorics, Computability and Logic, C. S. Calude, M. J. Dinneen, and S. Sburlan, Eds., Discrete Mathematics and Theoretical Computer Science, pp. 97-114, Springer, 2001.

[3] P. Hájek, Metamathematics of Fuzzy Logic, vol. 4 of Trends in Logic, Kluwer Academic Publishers, 1998.

[4] A. Iorgulescu, "Iséki algebras. Connection with BL algebras," Soft Computing, vol. 8, no. 7, pp. 449-463, 2004.

[5] A. Iorgulescu, "Some direct ascendents of Wajsberg and MV algebras," Scientiae Mathematicae Japonicae, vol. 57, no. 3, pp. 583-647, 2003.

[6] P. Agliano and F. Montagna, "Varieties of BL-algebras. I. General properties," Journal of Pure and Applied Algebra, vol. 181, no. 2-3, pp. 105-129, 2003.

[7] A. di Nola, G. Georgescu, and A. Iorgulescu, "Pseudo-BL algebras. I," Multiple-Valued Logic, vol. 8, no. 5-6, pp. 673-714, 2002.

[8] F. Esteva and L. Godo, "Monoidal t-norm based logic: towards a logic for left-continuous t-norms," Fuzzy Sets and Systems, vol. 124, no. 3, pp. 271-288, 2001.

[9] P. Hájek, "Fleas and fuzzy logic," Journal of Multiple-Valued Logic and Soft Computing, vol. 11, no. 1-2, pp. 137-152, 2005.

[10] A. Iorgulescu, "On pseudo-BCK algebras and porims," Scientiae Mathematicae Japonicae, vol. 10, pp. 293-305, 2004.

[11] J. Kühr, Pseudo-BCK-algebras and related structres [Ph.D. thesis], Univerzita Palackého v Olomouci, 2007. 


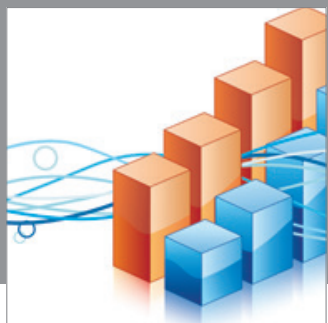

Advances in

Operations Research

mansans

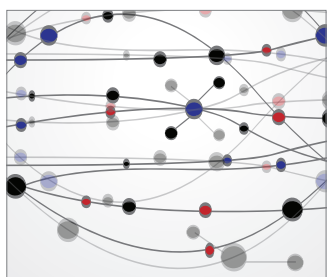

The Scientific World Journal
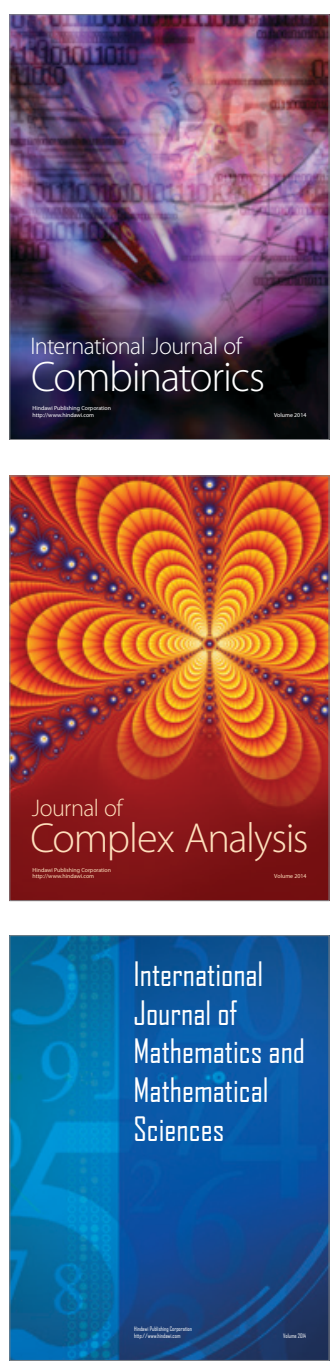
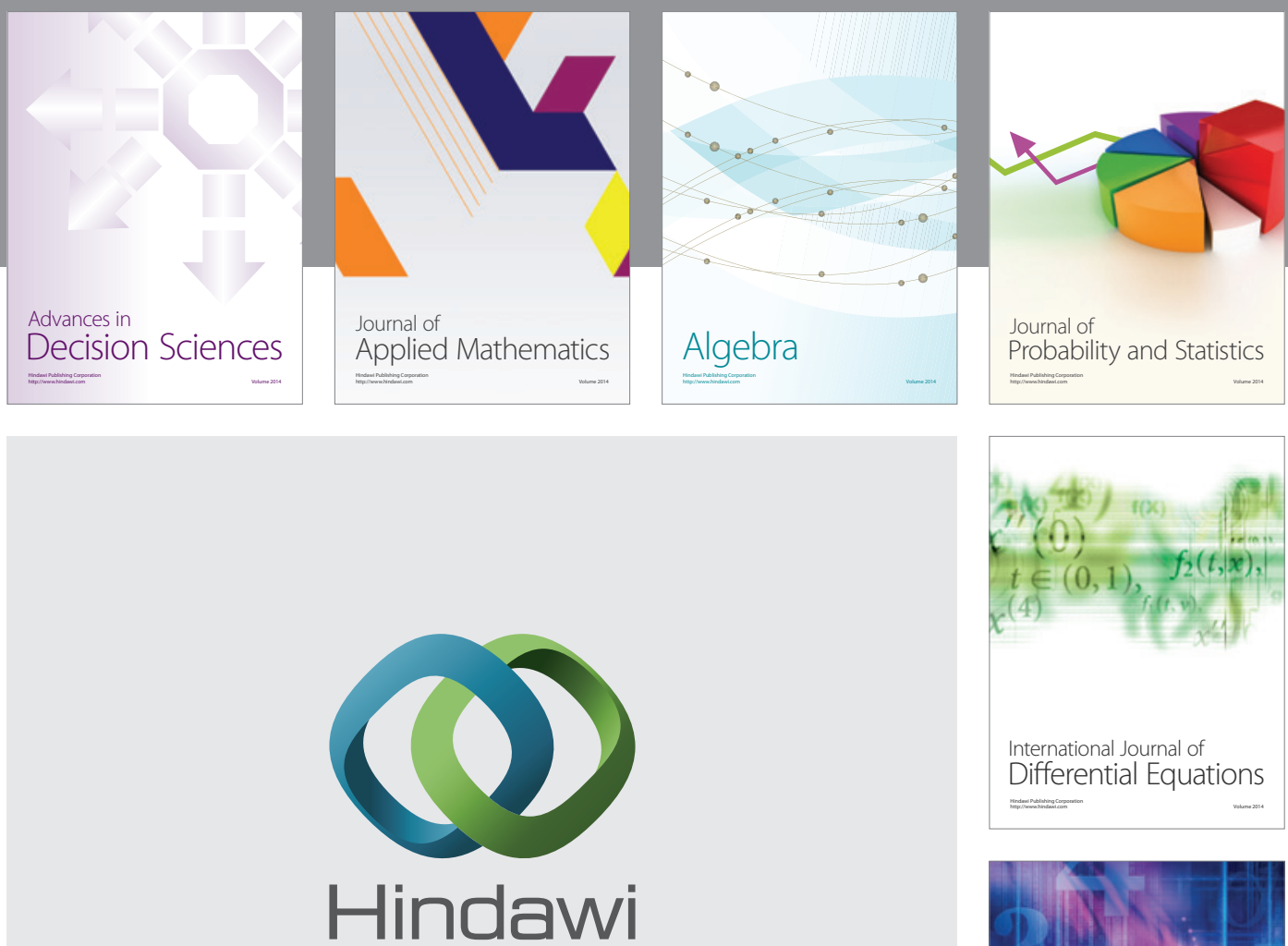

Submit your manuscripts at http://www.hindawi.com
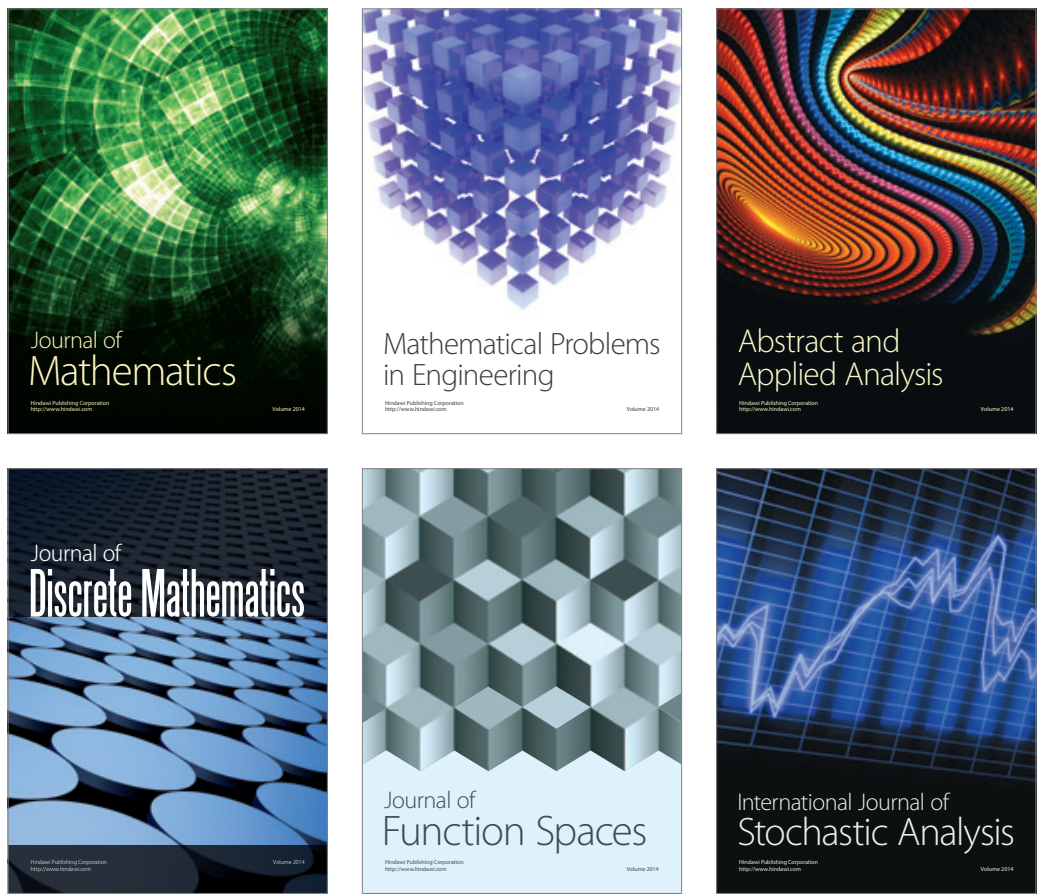

Journal of

Function Spaces

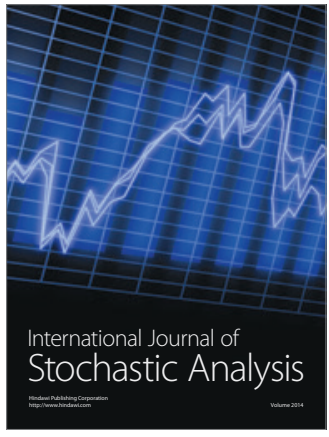

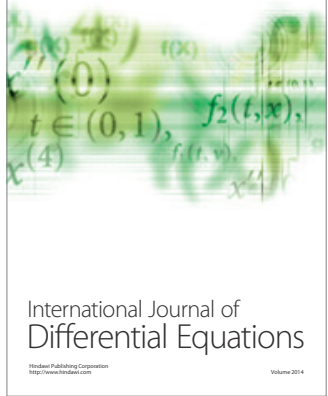
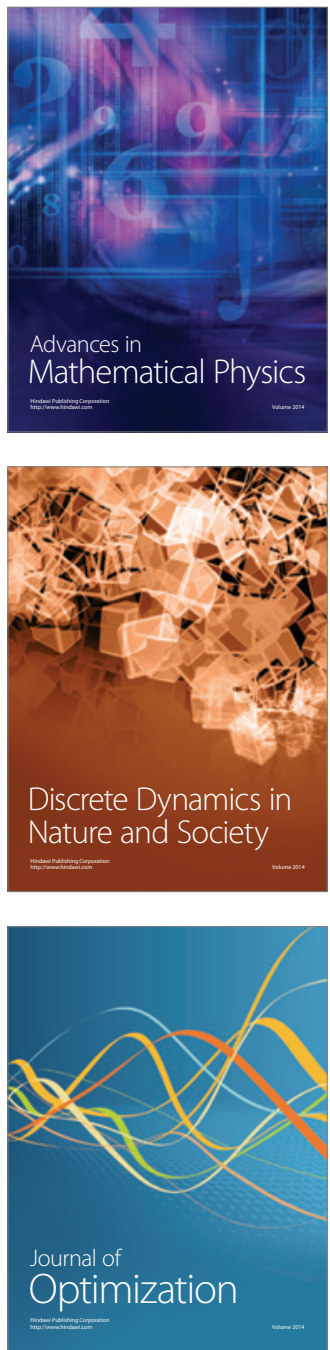